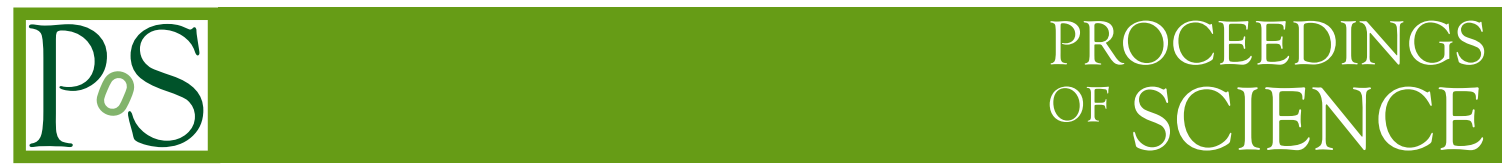

\title{
ATLAS Open Data: Data visualisation and educational physics analysis to re-discover the Higgs boson
}

\section{Meirin Oan Evans, on behalf of the ATLAS Collaboration ${ }^{a, *}$}

${ }^{a}$ School of Mathematical and Physical Sciences, University of Sussex, Falmer, Brighton, Great Britain

E-mail: meirin.oan.evans@cern.ch

\begin{abstract}
ATLAS Open Data aims to introduce students to particle-physics analysis without an initial exposure to the coding behind, with tools for interactive data visualisation. Web-based resources such as the "Histogram Analyser" are easy to use, yet informative. Students intrigued by the physics can then build up to coding an analysis by running Jupyter notebooks out of the box. Such notebooks invite them to read some short pieces of code, but without asking them to write any, just yet. With the use of these tools and documentation, students can be guided to find the Higgs boson with only their mouse!
\end{abstract}

The Eighth Annual Conference on Large Hadron Collider Physics-LHCP2020

25-30 May, 2020

online

${ }^{*}$ Speaker 


\section{Data visualisation and educational physics analysis to re-discover the Higgs boson}

ATLAS [1] Open Data provides data and tools for education. Tools include analysis examples, frameworks and virtual machines. Coming with these tools is extensive documentation (Figure 1). ATLAS Open Data are aimed primarily at university students. Some of the activities that students can perform are illustrated in Figure 2. These proceedings focus on hunting for the Higgs boson.

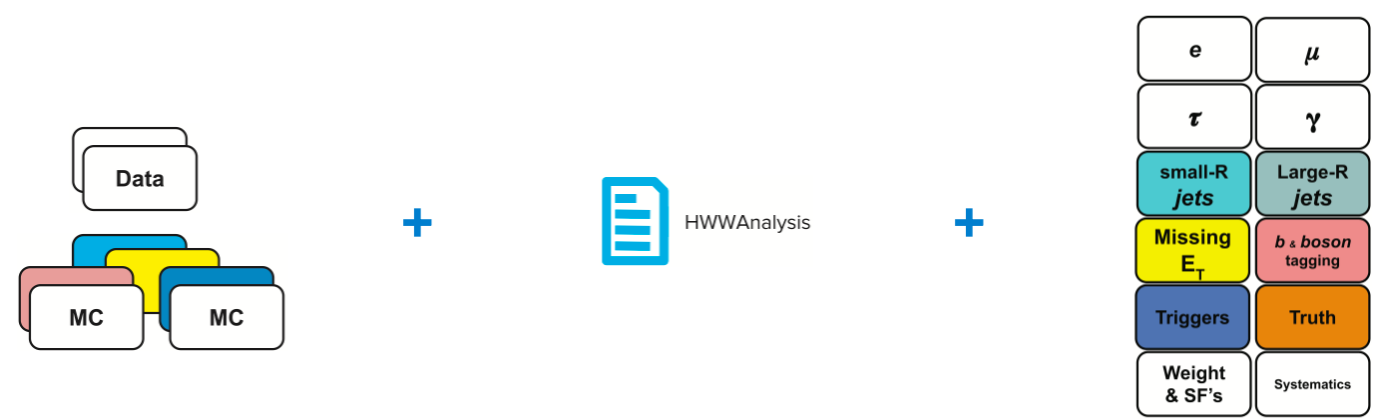

Figure 1: The ATLAS Open Data project provides datasets (both real and simulated), along with tools and documentation. Tools include example analyses and software that are presented here.
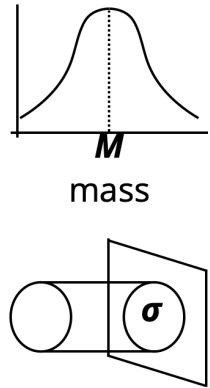

cross-section

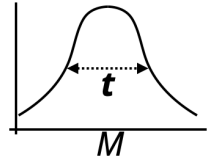

lifetime

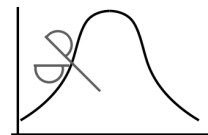

modify cuts

Figure 2: Some of the activities that students can perform with ATLAS Open Data. Examples include measuring particle masses, lifetimes and cross sections, and seeing what happens when they modify cuts.

\section{Histogram Analyser}

The first data visualisation tool presented is the Histogram Analyser [2], shown in Figure 3. The Histogram Analyser was initially made for $8 \mathrm{TeV}$ ATLAS Open Data [3], and has been updated with new $13 \mathrm{TeV}$ data [4]. The Histogram Analyser is a fast, web-based application.

\section{Jupyter notebooks}

Building towards analysis from the Histogram Analyser, the next tools are Jupyter [5] notebooks, where students can recreate the discovery of the Higgs boson by running analyses out of the box. The analysis presented in Figure 4 recreates the famous $H \rightarrow \gamma \gamma$ peak [6], with a bump around $125 \mathrm{GeV}$ [7]. ATLAS Open Data notebooks cover a variety of topics written in multiple programming languages. Notebooks are complemented by Feynman diagrams such as those shown in Figure 5. 

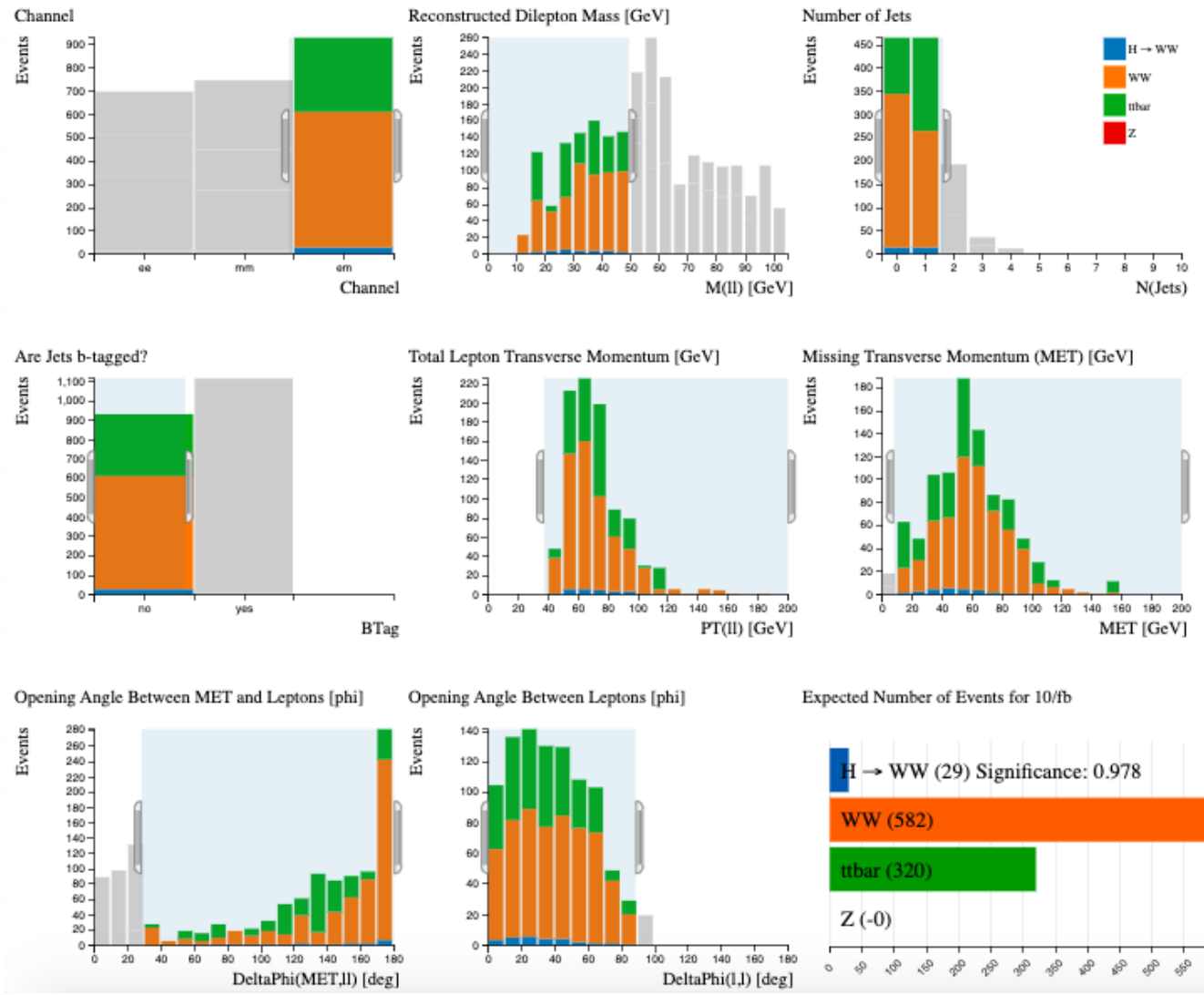

Expected Number of Events for 10/fb

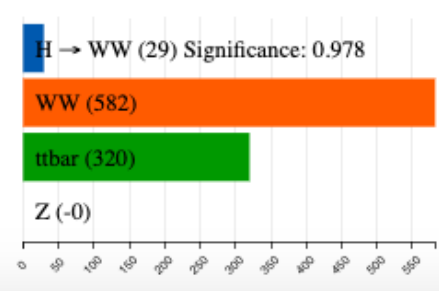

Figure 3: Screenshot of the $13 \mathrm{TeV}$ ATLAS Open Data Histogram Analyser. This analysis searches for the Higgs boson in the $H \rightarrow W^{+} W^{-} \rightarrow \ell^{+} v \ell^{-} v$ channel. Students learn by making selections with their mouse.

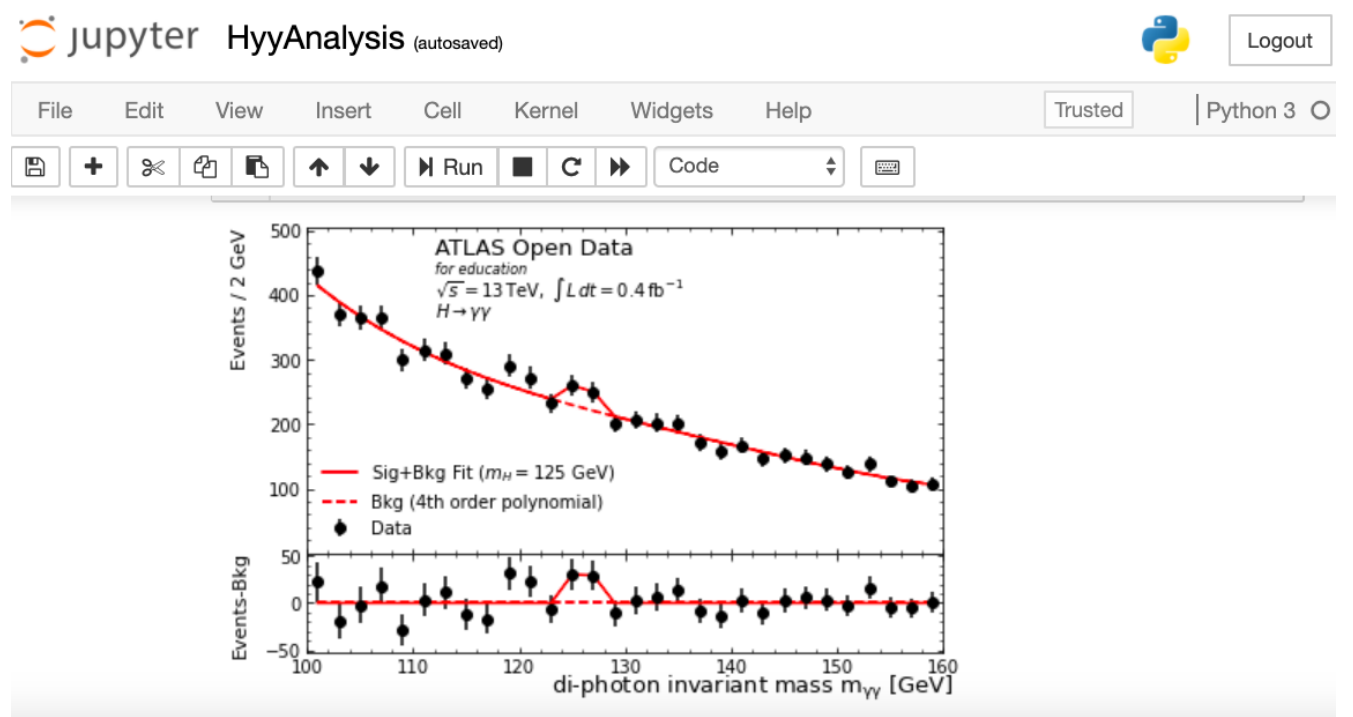

Figure 4: Screenshot of the Jupyter notebook "How to re-discover the Higgs boson", which takes about 5 minutes to complete. It recreates the ATLAS Higgs boson discovery, in the $H \rightarrow \gamma \gamma$ channel [6], by plotting the di-photon invariant mass. Included are a data fit, background fit and a "data minus background" subplot. 


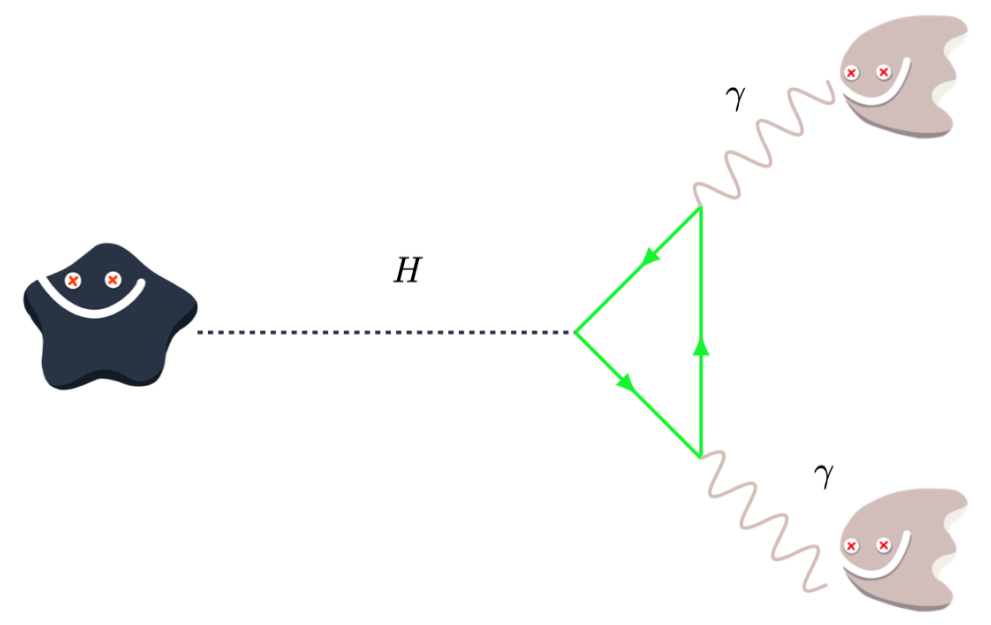

Figure 5: Feynman diagram in the $H \rightarrow \gamma \gamma$ Jupyter notebook, using pictures from the Particle Zoo [8].

\section{Conclusion}

With the ATLAS Open Data Histogram Analyser and Jupyter notebooks, students can carry out physics analyses in a visual manner to re-discover the Higgs boson for themselves!

\section{References}

[1] ATLAS Collaboration, The ATLAS Experiment at the CERN Large Hadron Collider, JINST 3 (2008) S08003.

[2] ATLAS Collaboration, 2020, Histogram Analyser, ATLAS Open Data 13 TeV Documentation, opendata.atlas.cern/release/2020/documentation/visualization/the_display_histograms_13TeV.html (Accessed on 1 September, 2020).

[3] ATLAS Collaboration, Review of ATLAS Open Data $8 \mathrm{TeV}$ datasets, tools and activities, ATL-OREACH-PUB-2018-001, https://cds.cern.ch/record/2624572 (2018).

[4] ATLAS Collaboration, Review of the $13 \mathrm{TeV}$ ATLAS Open Data release, ATL-OREACH-PUB2020-001, https://cds.cern.ch/record/2707171 (2020).

[5] Project Jupyter, 2020, https://jupyter.org (Accessed on 1 September, 2020).

[6] ATLAS Collaboration, Observation of a new particle in the search for the Standard Model Higgs boson with the ATLAS detector at the LHC, Phys. Lett. B 716 (2012) 1 [hep-ex/1207.7214v2].

[7] ATLAS Collaboration, 2020, How to rediscover the Higgs, Notebooks Collection Open Data, https://mybinder.org/v2/gh/atlas-outreach-data-tools/notebooks-collectionopendata/master?filepath=13-TeV-examples/uproot_python/HyyAnalysis.ipynb (Accessed on 1 September, 2020).

[8] The Particle Zoo, 2020, https://www.particlezoo.net/ (Accessed on 1 September, 2020). 